\title{
Editorial
}

\section{Empirical Research on Pedestrians' Behavior and Crowd Dynamics}

\author{
Milad Haghani (D), ${ }^{1}$ Nikolai W. F. Bode, ${ }^{2}$ Maik Boltes, ${ }^{3}$ Alessandro Corbetta, ${ }^{4}$ \\ and Emiliano Cristiani ${ }^{5}$ \\ ${ }^{1}$ Institute of Transport and Logistics Studies, The University of Sydney Business School, The University of Sydney, NSW, Australia \\ ${ }^{2}$ Department of Engineering Mathematics, University of Bristol, Bristol, UK \\ ${ }^{3}$ Forschungszentrum Jülich, Jülich, Germany \\ ${ }^{4}$ Department of Applied Physics, Eindhoven University of Technology, Eindhoven, Netherlands \\ ${ }^{5}$ Istituto per le Applicazioni del Calcolo "M. Picone", National Research Council of Italy, Rome, Italy \\ Correspondence should be addressed to Milad Haghani; milad.haghani@sydney.edu.au
}

Received 29 July 2019; Accepted 1 August 2019; Published 12 November 2019

Copyright ( 2019 Milad Haghani et al. This is an open access article distributed under the Creative Commons Attribution License, which permits unrestricted use, distribution, and reproduction in any medium, provided the original work is properly cited.

The increasing urban population around the world means that transportation hubs and large-scale buildings host a rising number of users and occupants; and mass gatherings are more frequent than ever before. Therefore, the monitoring, design and management of crowded spaces in which people move on foot is paramount for urban planners, event organisers and safety authorities. A scientific approach to these problems requires data and this special issue is thus aimed at encouraging and collecting empirical research into pedestrian behaviour and crowd dynamics.

Different research questions and application areas mean that pedestrian behavior and crowd dynamics are investigated across a broad range of spatial and temporal scales. The papers collected in this special issue demonstrate that methodological and technological developments now make it possible to cover spatial scales ranging from confined bottleneck scenarios to city quarters and temporal scales from minutes to days. Similarly, this special issue also highlights the diversity of questions that can be and are being investigated empirically, including assessments of pedestrian behaviour on stairs, when boarding trains and in the presence of people with mobility impairments, for example.

Empirical data on pedestrian behavior and crowd dynamics is not only useful to directly inform our understanding or to facilitate the development of monitoring methodologies, but it also helps to test the theory developed in this field. A substantial research effort has been directed at investigating pedestrian behavior and crowd dynamics theoretically using mathematical or computational models [1-3]. Empirical data is essential to link numerical simulation models to real-life crowd phenomena to ensure that they represent the real world and hence can be used for prediction, planning and policy making. Although numerical models are often formulated for descriptive capabilities, it is not clear how they can be reliably utilised for prescriptive purposes [4]. In addition, it is also essential to ascertain via experimental or observational data the validity of the hypotheses that are obtained from models.

This special issue is in line with the increasing recognition that experimental and data driven studies of crowd dynamics have received in the last few years [5]. We hope that the publication of this special issue can further contribute to the harmonisation of research efforts in this field [6] and to address some of the research gaps outlined earlier. Ten papers were accepted for final publication and are briefly introduced below.

Sparnaaij et al. contribute a paper on the topic of calibration, as a topic that they describe "has received relatively little attention within the field of pedestrian modelling". Their paper highlights the fact that most methodologies proposed for calibration concern only a single movement type or a single metric and that this makes the applicability of such calibration procedures questionable. In addressing this topic, they pursue two goals: "(1) determine the effect of the choice of movement base cases, metrics, and density levels on the calibration results and (2) to develop a multiple-objective calibration approach". Their proposed calibration methodology is based on multiple normalized metrics that are combined as a weighted sum. 
Two articles in this special issue are concerned with the flow of dense crowds through narrow bottlenecks. Seriani et al. conduct their study on the effect of the ratio between passengers boarding and alighting at metro stations. They utilise an innovative experimental method where, using a mock-up physical model of a train vehicle, they conduct a series of experiments under different load conditions. The paper by Geoerg et al. addresses how the presence of people with impaired mobility within the crowd affects the movement of pedestrians through narrow bottlenecks. In doing that, the authors use a heterogenous population of pedestrians in their corridor and bottleneck experiments. Their sample of participants includes a mixture of individuals with and without mobility impairment. In this case, the mobility impaired individuals are all wheelchair users. The authors show that the flow-density relation is strongly affected by the presence of participants with disabilities.

The contribution by Ye et al. focuses on the dynamics of pedestrian interactions in a relevant and ubiquitous scenario: staircases. The authors, employing real-life data collected in a metro station in Shanghai, China, investigate the occurrence of overtaking and avoidance behaviors at different levels of services. The analysis, which considers ascending and descending flows, employs macroscopic (frequencies, densities) and microscopic (distances) indicators to characterize critical densities at which these phenomena appear, are maximum, and disappear.

The paper submitted by Hasani et al. looks at a different angle, the safety of pedestrians when crossing roads at signalised intersections. Using multiple data sources and data mining techniques, the authors adopted automated video processing methods to identify signalized intersections with highest risk for walking and bicycling for the case study of the city of San Diego, California, USA.

Railway stations can be used for public transportation, work, commerce, and leisure. Jeong et al. present a method for identifying the indoor space characteristics by clustering trajectories of visitors according to the derived distribution of density, velocity and direction angle. Six categories emerge from the clustering indicating the quality of utilization efficiency, the level of mobility, and the level of comfort. Jeong et al. present a case study for the Samseong station in Seoul, South Korea.

The contribution by Wang et al. highlights the large spatial scales at which pedestrians' movements are being considered empirically by presenting a novel method for integrating localised automated pedestrian counts into pedestrian density maps that span entire city quarters. The substantial improvement in accuracy of these maps compared to existing approaches suggests that real-time monitoring of pedestrian traffic at the city level may soon become technically feasible.

The paper by Gao et al. provides a pedestrian simulation tool which combines the visibility graph at the strategic level, multi-stage route choice scheme at the tactical level, and a variant of the social force algorithm at the operational level. The resulting model can generate a collision-free global route including a local route planning model based on estimated passing time, thus incorporating the tendency of individuals to avoid congestion. The case of passengers passing through a series of gates in Beijing Subway is investigated in detail, comparing simulated and real data.

We, the guest editors, contribute a systematic review of three terms that have been discussed controversially in the pedestrian and crowd dynamics literature: panic, irrationality and herding. We show that there is no consensus in the definition and use of the former two terms, but that there is the nucleus of an empirical investigation of the broad concept often described as herding. We suggest that instead of using the vague concepts of panic, irrationality and herding, tangible and quantifiable terms be used to facilitate empirical and theoretical progress in the field.

In line with the recommendation of the review article by the guest editors, Haghani and Sarvi contribute a paper that aims to operationalise the concept of rationality in collective escape by attributing rationality to 'behavioural optimality'. Haghani and Sarvi suggest that such a shift in perspective can make it possible to measure and quantify the ill-defined notion of rationality in crowd dynamics. The authors make a distinction between behaviour optimality at the level of individuals and the collective level. They also emphasise on the necessity of establishing proper reference points at each level and demonstrate possible reference points for analysis of behaviour optimality using experimental data.

From this brief discussion of the contributions selected for this special issue, it is clear that pedestrian behavior and crowd dynamics are studied empirically across a wide range of spatial and temporal scales. Future work will continue to drive the development of more detailed, longer lasting and further ranging empirical investigation. We hope this special issue provides a useful snapshot not only of what is currently feasible but also of future direction of empirical research on pedestrian behavior and crowd dynamics.

Milad Haghani
Nikolai W. F. Bode
Maik Boltes
Alessandro Corbetta
Emiliano Cristiani

\section{Conflicts of Interest}

The authors declare that there is no conflict of interest.

\section{References}

[1] D. C. Duives, W. Daamen, and S. P. Hoogendoorn, "State-of-theart crowd motion simulation models," Transportation Research Part C: Emerging Technologies, vol. 37, pp. 193-209, 2013.

[2] E. Ronchi, E. Kuligowski, and S. Gwynne, "Guest editorial: special issue on advances in evacuation modelling," Fire Technology, vol. 55, no. 2, pp. 387-389, 2019.

[3] M. Haghani and M. Sarvi, "Simulating pedestrian flow through narrow exits," Physics Letters A, vol. 383, pp. 110-120, 2019.

[4] E. Cristiani and D. Peri, "Robust design optimization for egressing pedestrians in unknown environments," Applied Mathematical Modelling, vol. 72, pp. 553-568, 2019.

[5] M. Haghani and M. Sarvi, "Crowd behaviour and motion: empirical methods," Transportation Research Part B: Methodological, vol. 107, pp. 253-294, 2018.

[6] J. Adrian, N. Bode, M. Amos et al., "A glossary for research on human crowd dynamics," Collective Dynamics, vol. 4, pp. 1-13, 2019. 


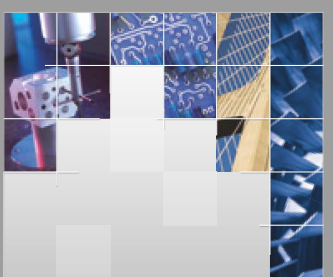

\section{Enfincering}
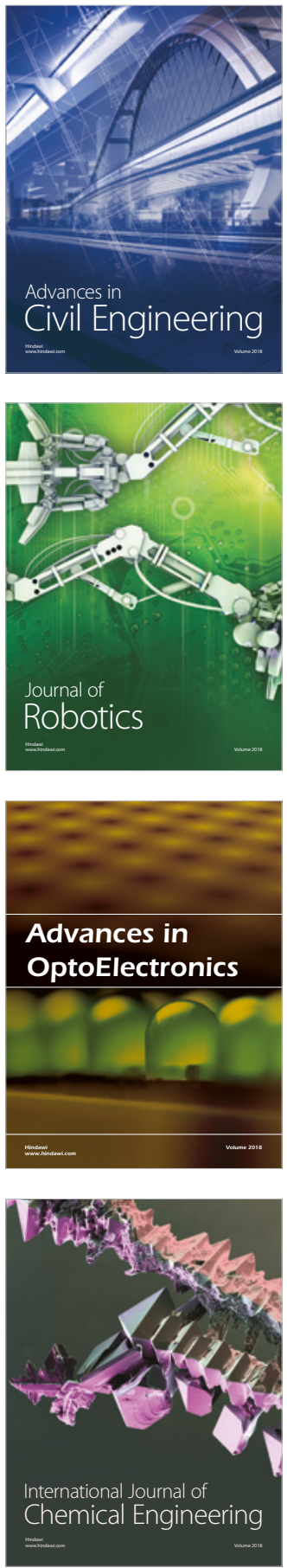

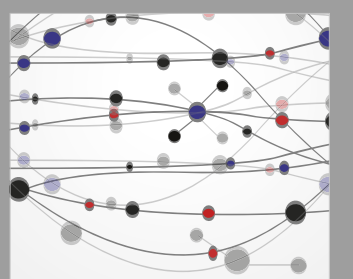

\section{Rotating \\ Machinery}

The Scientific World Journal

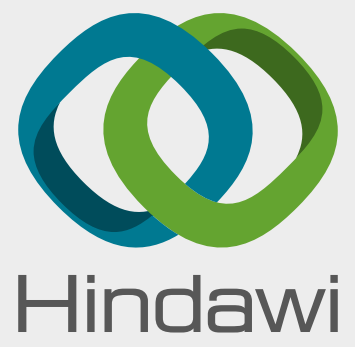

Submit your manuscripts at

www.hindawi.com
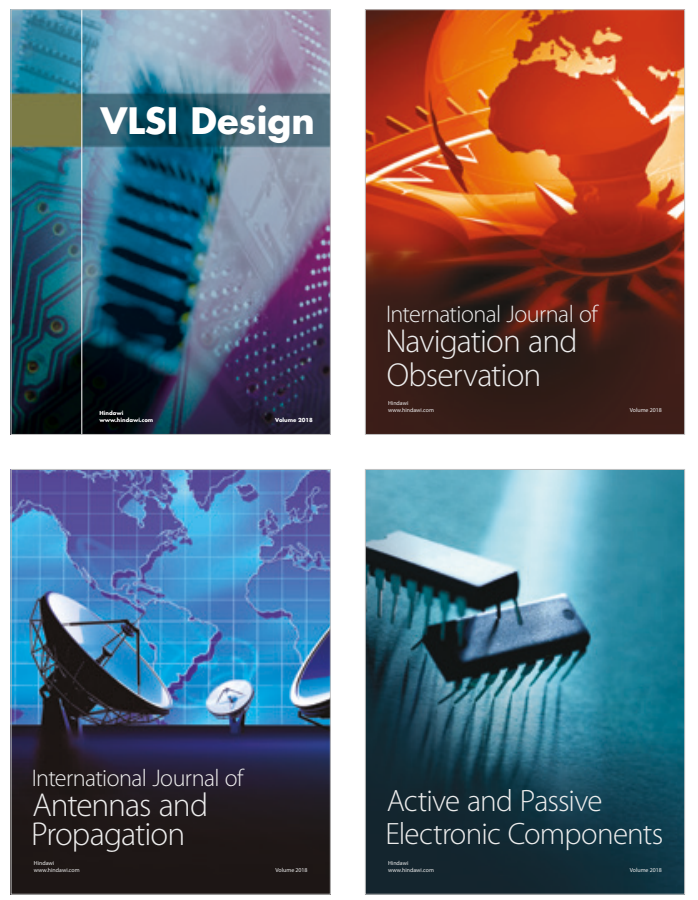
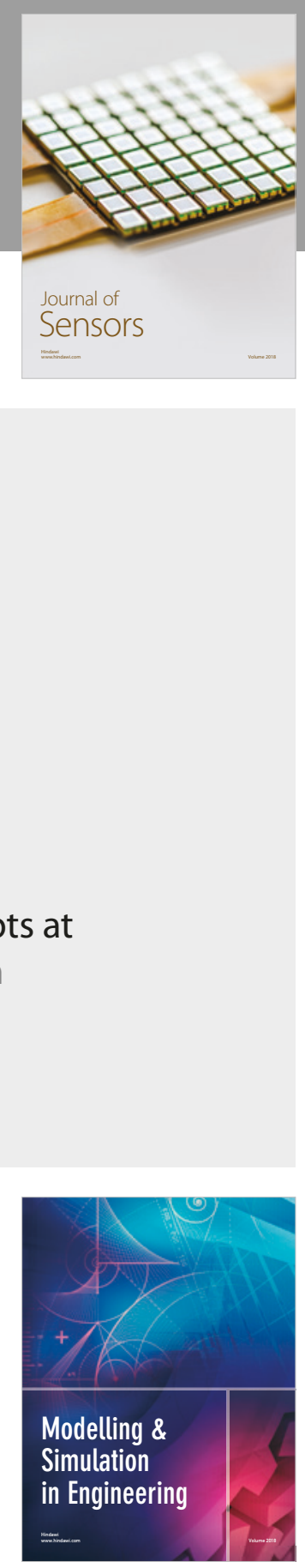

\section{Advances \\ Multimedia}
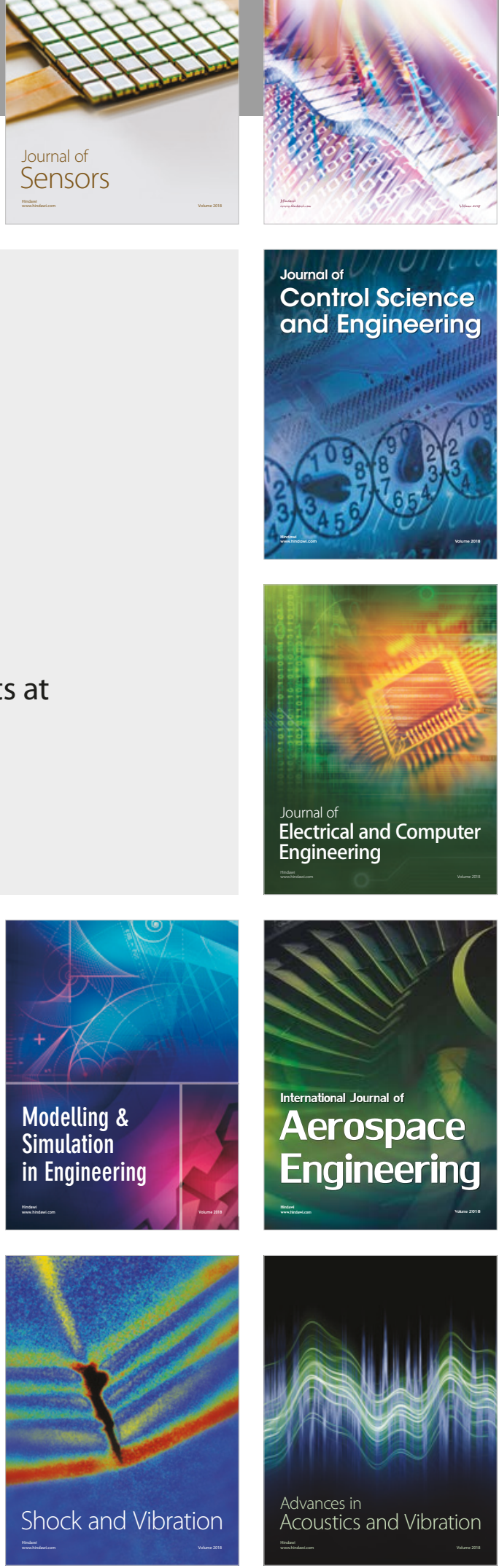